\title{
Study on Flows inside and outside an Air Diffuser for Membrane Bioreactor*
}

\author{
Fumihiro KIRA**, Shinsuke FURUNO**, \\ Kosuke HAYASHI***, Tomoyuki SAMPEI*** and Akio TOMIYAMA*** \\ **Mitsubishi Rayon Co., Ltd. \\ 4-1-2, Ushikawa-dohri, Toyohashi, Aichi, 440-8601, Japan \\ E-mail: kira_fu@mrc.co.jp \\ ${ }^{* * *}$ Graduate School of Engineering, Kobe University \\ 1-1 Rokkodai, Nada, Kobe, Hyogo, 657-8501, Japan
}

\begin{abstract}
Effects of the total gas flow rate on the water level in a diffuser pipe for a membrane bioreactor, the gas flow rate from each aeration hole and the bubble diameter are investigated. The diffuser has evenly positioned five aeration holes on the top and a larger hole on the bottom for introducing the liquid into the pipe. The gas flow rate from each aeration hole is measured by capturing generated bubbles. The water level and gas velocity inside the diffuser are computed by processing video images. The bubble diameter is calculated using the gas flow rate and the bubble generation frequency measured from the video images. The conclusions obtained are as follows: (1) the gas flow rate from the aeration hole depends on the water level inside the diffuser and becomes constant for all the holes as the total gas flow rate increases since the high total gas flow rate make the water level uniform, which results in a constant gas pressure in the diffuser, (2) the onset of slugging in the diffuser is well correlated in terms of the local gas velocity and the Mishima-Ishii's slugging model, (3) the increase in the total gas flow rate decreases the water level, causing suppression of the onset of slugging, (4) the diameter of aeration hole strongly affects the gas flow rate from each aeration hole and water level, and (5) the Davidson-Schuler correlation gives reasonable estimations of the bubble diameter, provided that the influence of slugging is not significant.
\end{abstract}

Key words: Air Diffuser, Membrane Bioreactor (MBR), Bubble, Slugging

\section{Introduction}

Membrane bioreactor (MBR) has been utilized in many wastewater treatment processors $^{(1)(3)}$. MBR consists of porous membranes for water filtration and air diffusers for supplying oxygen to microbes and removing suspended solids sticking to membrane surfaces. Since water processed by MBR is of high purity, the process does not require settling tanks. Because of this, the plant area can be reduced compared with conventional activated sludge processors. These advantages expand application of MBR, e.g., a sewage disposal plant, an industrial wastewater disposal plant and so on. However the energy consumption of MBR is large mainly due to the continuous aeration for oxygen supply and membrane cleaning ${ }^{(4)(5)}$. This makes the running costs higher than the conventional activated sludge processors. For the cost reduction, it is, therefore, very important to develop air diffusers, which can efficiently clean up membranes.

The air diffusers for MBR are mounted below the membranes. Horizontal circular pipes with multiple aeration holes have been used in some MBRs ${ }^{(6)(7)}$. Since wastewater entering into the pipes through the aeration holes dries and fills up the holes, the pipes also 
have openings for introducing wastewater into the pipe to rewet the dried sludge ${ }^{(8)}$. The same gas flow rate from all the aeration holes is desired to uniformly clean the membranes. The total gas flow rate, hole diameter and liquid flow inside the pipe may affect the gas flow rate from each hole of the pipe of the air diffuser. However our knowledge on their effects on the flow rate from each hole is insufficient since the presence of the multiple aeration holes and the opening makes the flow inside the pipe complex and few studies have been conducted on the flow rate from each hole.

Effects of the total gas flow rate and hole diameter on the gas flow rate from each aeration hole of a diffuser pipe and water level in the pipe were therefore investigated in this study. Since the bubble diameter is one of the important variables affecting membrane-cleaning efficiency, the bubble diameter was also measured and the applicability of an available correlation for bubble diameter was examined.

\section{Experimental}

Figure 1 shows the experimental setup. The test section was the horizontal pipe mounted on the rectangular water tank and was made of transparent acrylic resin. Air and tap water at atmospheric pressure were used for the gas and liquid phases, respectively. Their temperatures were measured at the beginning and the end of each measurement by using a thermometer (Netsuken, SN3000, accuracy: $\pm 0.5{ }^{\circ} \mathrm{C}$ ) and were kept to within $23 \pm 1$ and $13 \pm 1{ }^{\circ} \mathrm{C}$, respectively, in all the test conditions. The water level in the tank was 390 $\mathrm{mm}$. The distance between the free surface and the pipe axis was $280 \mathrm{~mm}$. Tap water was supplied to the tank at a constant flow rate and the excess water flowed over the vertical separation board to keep the water level constant. The gas phase supplied from the compressor

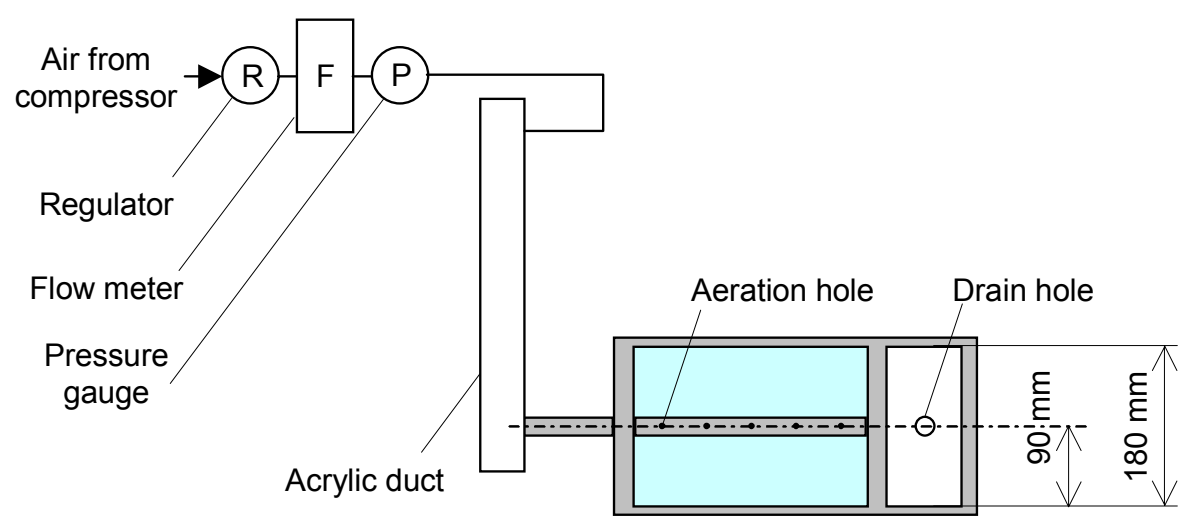

(a) Top view

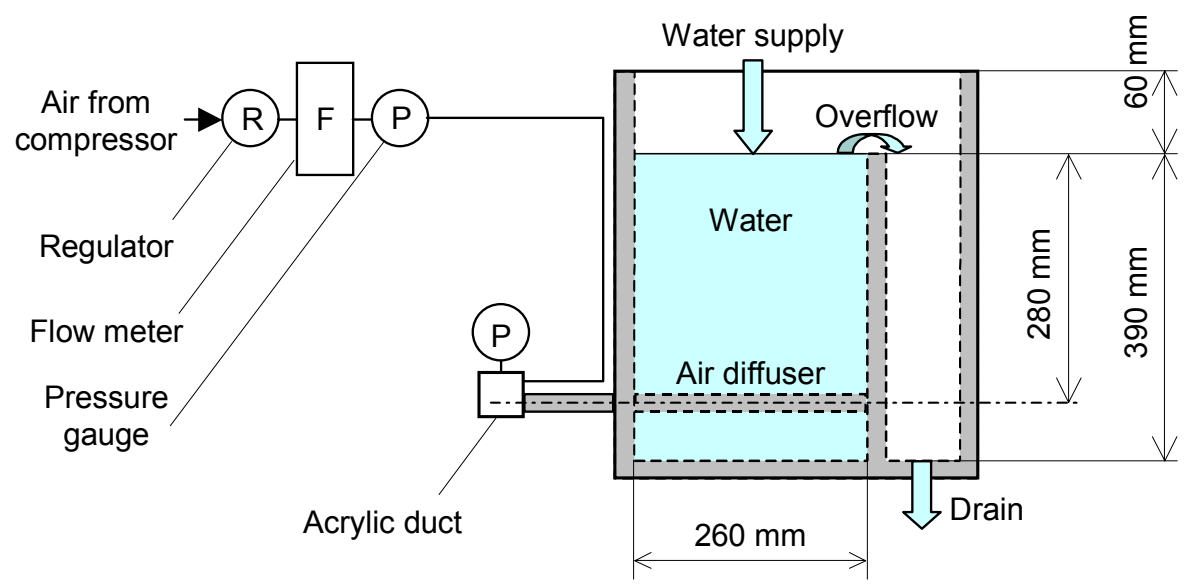

(b) Side view

Fig. 1 Experimental setup 

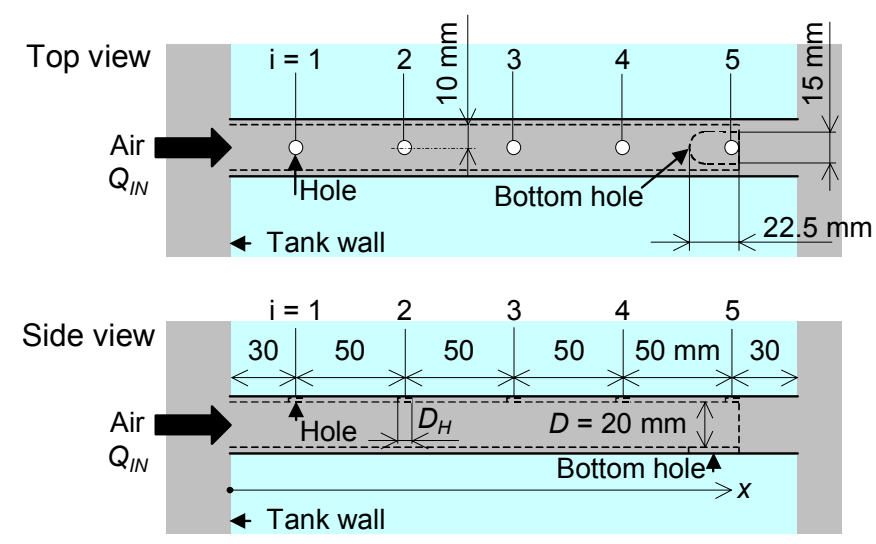

Fig. 2 Air diffuser

(Hitachi, SRL-2) flowed into the test section through the regulator (Nihon-seiki, BN-3RT5, 0-1.0 MPa), the flowmeter (Nippon flow cell, SPO-4, $1.0 \times 10^{-4}-1.0 \times 10^{-3} \mathrm{~m}^{3} / \mathrm{s}$ ) and the horizontal acrylic duct. The gas pressure in the duct was measured using the pressure gauge (Migishita Seiki, CA311-211-5KP, 0-5.0 kPa) and was about $2.8 \mathrm{kPa}$ (gauge) in all the test conditions.

The test section is shown in Fig. 2. The inner diameter, $D$, and the length of the pipe were $20 \mathrm{~mm}$ and $230 \mathrm{~mm}(0 \leq x \leq 230 \mathrm{~mm})$, respectively. The pipe had evenly positioned five aeration holes $(i=1-5)$ of the diameter $D_{H}$ on its top and an opening on the bottom. The centers of the holes $i=1$ and 5 were $30 \mathrm{~mm}$ apart from the tank walls to reduce the wall effect on the bubble generation process. Three sizes of $D_{H}, 2,5$ and $8 \mathrm{~mm}$, were tested to investigate the effect of hole diameter on the flow rate from each hole and water level in the pipe. The shape of the bottom hole projected on the horizontal plane was a combination of a half circle of $7.5 \mathrm{~mm}$ in radius and a square of $15 \mathrm{~mm}$ in width. The air was introduced from the left side at constant volume flow rates, $Q_{I N}$, and flowed out through the aeration holes. The values of $Q_{I N}$ tested were $3.0 \times 10^{-4}, 5.0 \times 10^{-4}$ and $7.0 \times 10^{-4} \mathrm{~m}^{3} / \mathrm{s}$. The pipe geometry and the flow rates were determined by referring a typical operating condition of an actual $\mathrm{MBR}^{(8)}$. The reference condition is that of $D_{H}=5 \mathrm{~mm}$ and $Q_{I N}=5.0 \times 10^{-4} \mathrm{~m}^{3} / \mathrm{s}$.

Bubbles generated from the aeration hole $i$ were captured by using a graduated cylinder (volume: $5.0 \times 10^{-4} \mathrm{~m}^{3}$ ) to measure the gas flow rate, $Q_{i}$, at the hole $i$. The measurement was repeated 30 times at each $i$ to obtain an average flow rate. The flowmeter for measuring $Q_{I N}$ was first calibrated using the sum of $Q_{i}$. The uncertainty estimated at $95 \%$ confidence in measured $Q_{I N}$ was less than $0.67 \%$ in all the test conditions.

Successive 8-bit grayscale images of flows inside the pipe and bubbles generated from the aeration holes were taken by using a high-speed video camera (Redlake, MotionPro $\mathrm{X}-3$ ). The shatter speed and frame rate were $1.9 \mathrm{~ms}$ and $500 \mathrm{fps}$, respectively. Two reflex lamps were used for back illumination. From the ten-second images (5000 frames), the bubble generation frequency, $f_{B i}$, at each $i$ was measured by counting the number of bubble detachment from the hole. The uncertainty in $f_{B i}$ was estimated as $\pm 0.3 \mathrm{~s}^{-1}$. Since the shapes of generated bubbles were too distorted to accurately measure the bubble size from the image, the sphere-volume equivalent bubble diameter, $d_{i}$, was evaluated by

$$
d_{i}=\left(\frac{6 Q_{i}}{\pi f_{B i}}\right)^{1 / 3}
$$

The water level, $h$, in the pipe was obtained from the video images by using an image processing method. The algorithm of the image processing is as follows: 
(1) Subtracting a background image from all the successive images.

(2) Transforming the images into black and white (1-bit) images using a threshold based on the discriminant analysis ${ }^{(9)}$. The water level is detected as a narrow-band black region.

(3) Shrinking the black region by one pixel, then expanding it by one pixel to remove small-scale noises.

(4) Searching the pixel at which the bit changes from 0 to 1 along the vertical line, i.e., the water level, from the pipe bottom to the top.

(5) Correcting the water level by considering the reflection at the pipe surfaces.

The spatial resolution of images for the water level evaluation was $0.18 \mathrm{~mm} / \mathrm{pixel}$. The water level detected in this method is the minimum level. In fact, the water surface in binary image had 12 pixels $(=2.2 \mathrm{~mm})$ in the vertical direction in average. The pipe wall was hydrophilic and the water surface was lifted up about $1 \mathrm{~mm}$ near the pipe wall due to the surface tension force. Therefore the actual average water level might be higher than the measured one by $1.2 \mathrm{~mm}$ at the largest. The images for 20 -seconds duration were used, i.e., the total number of frames was 10,000 .

The gas velocity, $u_{G}$, in the horizontal direction inside the pipe was also calculated using the continuity equation and $h$. The surface was assumed to be flat in the direction perpendicular to the image of flow when calculating $u_{G}$.

\section{Results and Discussion}

\subsection{Gas flow rate from aeration hole and water level $\left(D_{H}=\mathbf{5} \mathbf{m m}\right)$}

Figure 3 shows gas flow rates, $Q_{i}$, measured for $D_{H}=5 \mathrm{~mm}$. At $Q_{I N}=3.0 \times 10^{-4} \mathrm{~m}^{3} / \mathrm{s}, Q_{i}$ monotonously decreases in the $x$ direction. Especially $Q_{5}$ is approximately zero. The magnitude of gradient of $Q_{i}$ with respect to $x$ becomes small as $Q_{I N}$ increases. The same gas flow rate from all the aeration holes is achieved at the highest $Q_{I N}$.

Figure 4 shows flows inside the pipe and bubbles generated from the aeration holes. The water level in the pipe increases as $Q_{I N}$ decreases. At $Q_{I N}=3.0 \times 10^{-4} \mathrm{~m}^{3} / \mathrm{s}$, the hole $i=5$ was almost always filled with water, and therefore, $Q_{5}$ was approximately zero. The gas and liquid phases in the pipe at the highest $Q_{I N}$ (Fig. 4 (a)) were well separated and there were small amplitude waves on the surface. The two phases at lower $Q_{I N}$ were also well separated at the instances in Figs. 4 (b) and (c), whereas liquid slugs were intermittently formed at these $Q_{I N}$.

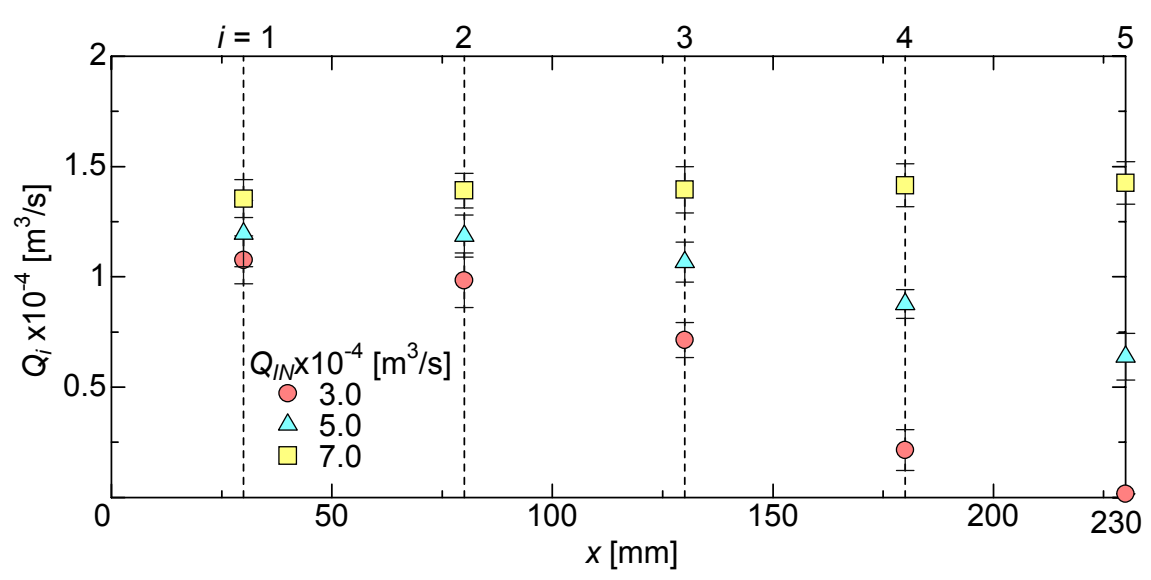

Fig. 3 Flow rates, $Q_{i}$, for $D_{H}=5 \mathrm{~mm}$ 


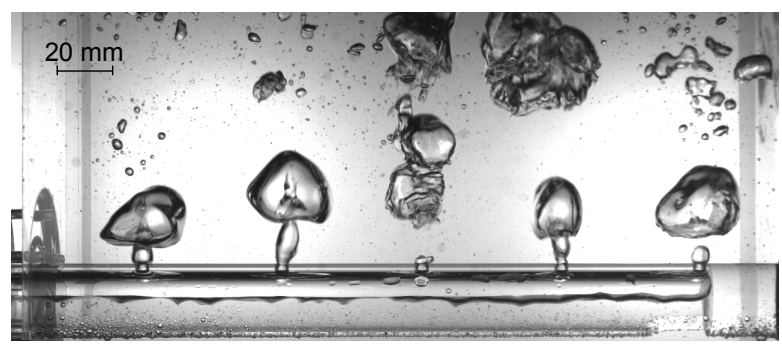

(a) $7.0 \times 10^{-4} \mathrm{~m}^{3} / \mathrm{s}$

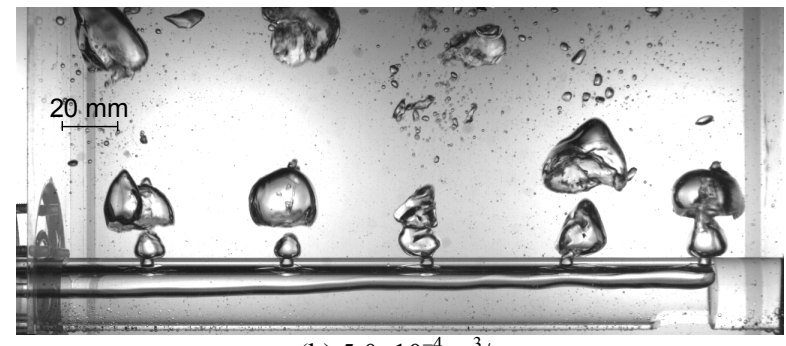

(b) $5.0 \times 10^{-4} \mathrm{~m}^{3} / \mathrm{s}$

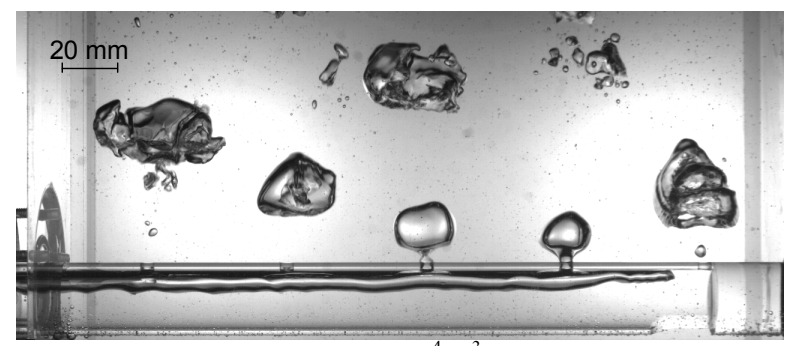

(c) $3.0 \times 10^{-4} \mathrm{~m}^{3} / \mathrm{s}$

Fig. 4 Flows inside the pipe and generated bubbles $\left(D_{H}=5 \mathrm{~mm}\right)$

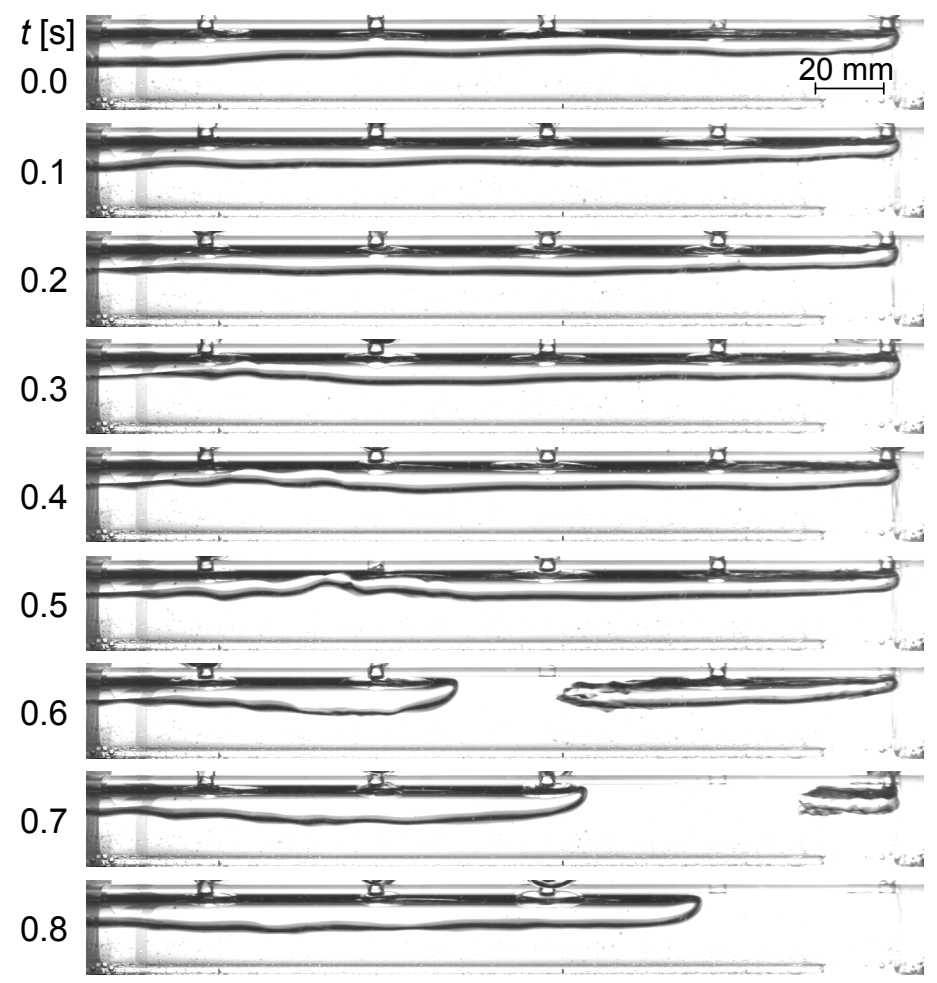

Fig. 5 Onset of slugging at $Q_{I N}=5.0 \times 10^{-3} \mathrm{~m}^{3} / \mathrm{s}$ and $D_{H}=5 \mathrm{~mm}$ 


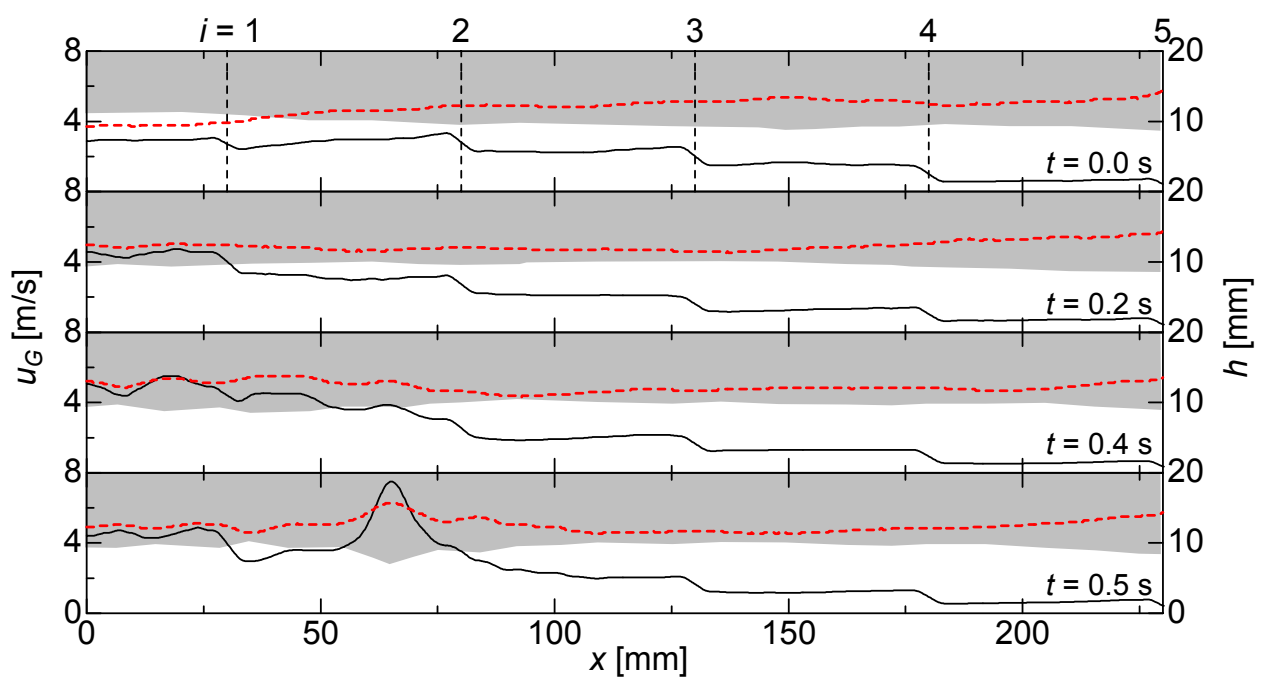

Fig. 6 Gas velocity (solid line) and water level (dashed red line) at the instants corresponding to those in

Fig. 5 . The inequality proposed by Mishima and Ishii is satisfied when $u_{G}$ is in the shaded region.

Figure 5 shows an example of the onset of slugging. At $t=0.0 \mathrm{~s}$, no liquid slugs are present. The water level between the inlet and $i=1$ becomes higher at $t=0.2 \mathrm{~s}$ and the wave crest grows in the region between $i=1$ and 2 for $t=0.3-0.5 \mathrm{~s}$. Then the liquid slug occupying the whole cross section is formed and runs toward the right wall for $t=0.6-0.8 \mathrm{~s}$. The frequencies of slugging were $1.2,0.70$ and $0.0 \mathrm{~Hz}$ at $Q_{I N}=3.0 \times 10^{-4}, 5.0 \times 10^{-4}$, and $7.0 \times 10^{-4} \mathrm{~m}^{3} / \mathrm{s}$, respectively, and therefore, the increase in $Q_{I N}$ suppresses the slugging. The liquid slug filling up the aeration holes causes the decrease in $Q_{i}$. This is one of the reasons why $Q_{i}$ decreases with increasing $i$ at the low and intermediate $Q_{I N}$. It is therefore important to know the instability criteria for the onset of slugging in the air diffuser.

Mishima and Ishii ${ }^{(10)}$ proposed the following model for the onset of slugging in horizontal circular pipes:

$$
u_{G}-u_{L} \geq 0.487 \sqrt{\frac{\rho_{L} g h_{G}}{\rho_{G}}}
$$

where $u_{L}$ is the liquid velocity, $\rho_{L}$ the liquid density, $\rho_{G}$ the gas density, $g$ the magnitude of gravity acceleration, $h_{G}$ the height of the gas phase $\left(h_{G}=D-h\right)$. Water levels and gas velocities at $t=0.0,0.2,0.4$ and $0.5 \mathrm{~s}$ are shown in Fig. 6 . The gas velocity satisfies the above inequality where the solid line is in the shaded region. At $t=0.0 \mathrm{~s}$, the gas velocity does not satisfy the inequality at any $x$. For $t \leq 0.1 \mathrm{~s}$, no liquid slugs grow. Then, $u_{G}$ exceeds the critical value in $x \leq 30 \mathrm{~mm}$ at $t=0.2 \mathrm{~s}$ and the slug grows from that region. Not only for this case but also for the other $Q_{I N}$, Eq. (2) gives good predictions for the onset of slugging in the diffuser.

The time-averaged $h$ is shown in Fig. 7. The water level at $Q_{I N}=3.0 \times 10^{-4} \mathrm{~m}^{3} / \mathrm{s}$ is higher than the pipe axis and increases with $x$. The water level decreases with increasing $Q_{I N}$, and is nearly flat at $Q_{I N}=7.0 \times 10^{-4} \mathrm{~m}^{3} / \mathrm{s}$. As can be understood from the distributions of $Q_{i}$ and $h$ shown in Figs. 3 and 7, $Q_{i}$ depends on $h$, i.e., the increase in $h$ decreases $Q_{i}$. To make clear the effect of $h$ on $Q_{i}, Q_{i}$ was also measured using a pipe without the bottom hole. Figure 8 shows a flow in the pipe without the bottom hole at $Q_{I N}=5.0 \times 10^{-4} \mathrm{~m}^{3} / \mathrm{s}$. Even without the bottom hole, small amount of water entered into the pipe through the aeration holes when bubbles detached. The water level, however, is much lower than that shown in Fig. 4 (b). The flow rate without the bottom hole is approximately the same for all the aeration holes, whereas that with the bottom hole decreases as $x$ increases as shown in Fig. 9. The same 
flow rate from each hole of the pipe without the bottom hole indicates that the gas pressure in the pipe is about the same at all $i$. In the case with the bottom hole, water inside the pipe rises to a level, at which the gas pressure balances with the static pressure of water at the surface. Therefore high $h$ corresponds to low gas pressure, and $Q_{i}$ decreases as $h$ increases.

Let us discuss the effects of $Q_{I N}$ on $Q_{i}$ and $h$ from the point of view of pressure drops at the aeration holes. Applying an orifice equation to the flow rate from each aeration hole yields $Q_{i}^{2}=k^{2}\left|P_{G i}-P_{B i}\right|$, where $P_{G i}$ is the pressure in the pipe at $i, P_{B i}$ the pressure in a bubble attached to the hole $i$, and $k$ the orifice coefficient. Terasaka and Tsuge ${ }^{(11)}$ measured bubbles generated from a single orifice and proposed a correlation for the orifice coefficient, $k=C_{k} D_{H}{ }^{2}$, where $C_{k}=0.813 \mathrm{~m}^{3 / 2} / \mathrm{kg}^{1 / 2}$. By assuming that $k$ is correlated in terms of $D_{H}$, the ratio of pressure drops, $\left|P_{G i}-P_{B i}\right| /\left|P_{G j}-P_{B j}\right|$, between holes $i$ and $j$ is given as $Q_{i}{ }^{2} / Q_{j}{ }^{2}$. For instance, $\left|P_{G 3}-P_{B 3}\right|$ at $Q_{I N}=7.0 \times 10^{-4} \mathrm{~m}^{3} / \mathrm{s}$ is 3.8 times higher than that at $Q_{I N}$ $=3.0 \times 10^{-4} \mathrm{~m}^{3} / \mathrm{s}$. This increase in pressure drop with $Q_{I N}$ makes $P_{G i}$ uniform in the $x$ direction, which results in uniform distributions of $h$ and $Q_{i}$ at high $Q_{I N}$.

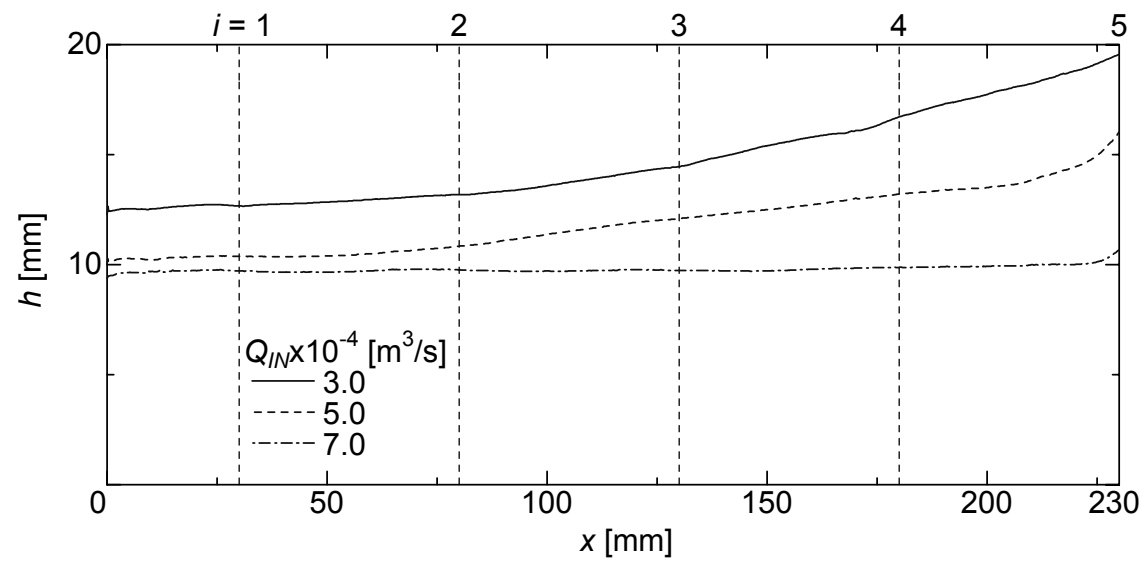

Fig. 7 Water level for $D_{H}=5 \mathrm{~mm}$

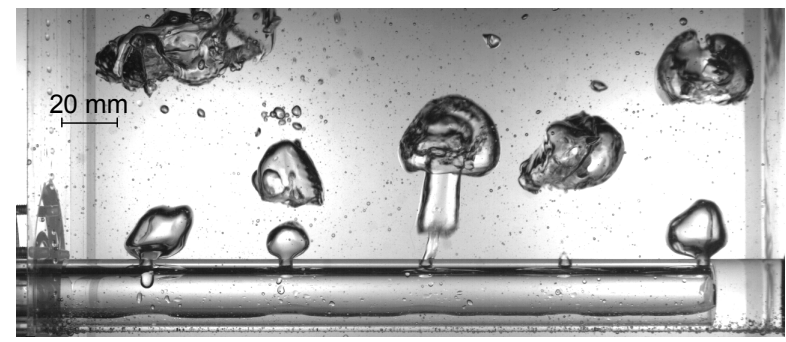

Fig. 8 Flow inside and outside pipe without bottom hole $\left(D_{H}=5 \mathrm{~mm}\right)$

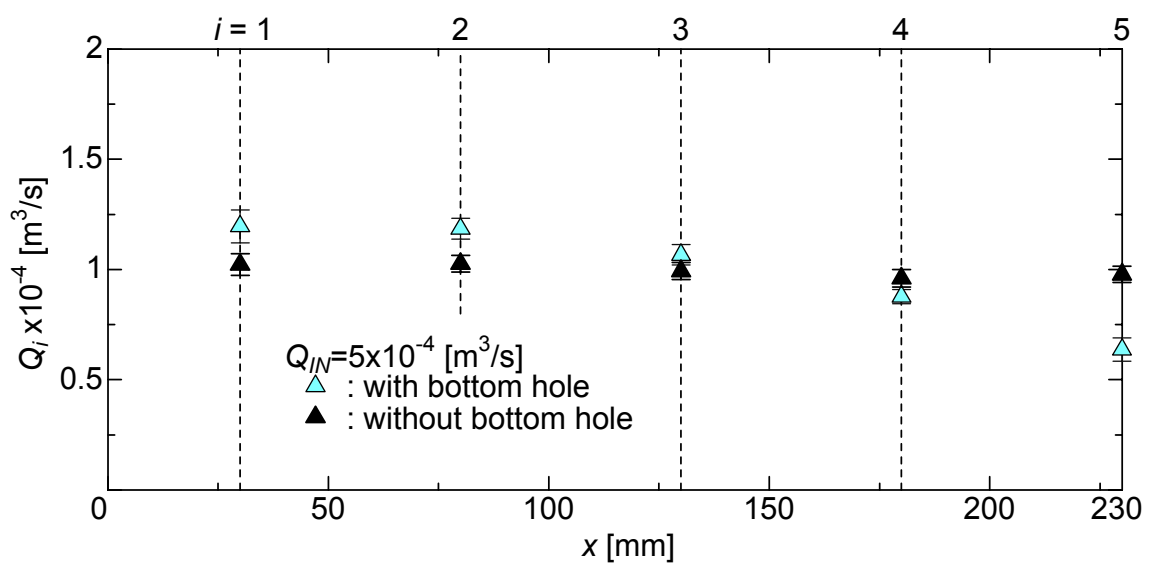

Fig. 9 Effect of the presence of bottom hole on flow rate $Q_{i}\left(D_{H}=5 \mathrm{~mm}\right)$ 


\subsection{Evaluation of bubble diameter}

The bubble generation frequency for $D_{H}=5 \mathrm{~mm}$ is shown in Fig. 10 (a). Similar to $Q_{i}$, $f_{B i}$ decreases with increasing $i$ at the low and intermediate $Q_{I N}$, whereas it is approximately constant for all $i$ at the highest $Q_{I N}$. The bubble diameter is shown in Fig. 10 (b). The trend of $d_{i}$ is the same as that of $Q_{i}$. However the influence of $Q_{I N}$ on $d_{i}$ is much smaller compared with that on $Q_{i}$ and $f_{B i}$, e.g., $Q_{5}\left(7.0 \times 10^{-4}\right) / Q_{5}\left(3.0 \times 10^{-4}\right)=85.4, f_{B 5}\left(7.0 \times 10^{-4}\right) / f_{B 5}\left(3.0 \times 10^{-4}\right)=$ 14.0 and $d_{5}\left(7.0 \times 10^{-4}\right) / d_{5}\left(3.0 \times 10^{-4}\right)=1.83$, where the value in the parentheses denotes the value of $Q_{I N}$. The bubble diameter is approximately $25 \mathrm{~mm}$ except for $i=4$ and 5 at $Q_{I N}=$ $3.0 \times 10^{-4}$ and $5.0 \times 10^{-4} \mathrm{~m}^{3} / \mathrm{s}$. Slugging is responsible for the exception: the intermittent liquid slug disturbs the bubble generation process at these holes and bubbles stop growing to detach before becoming large.

Since bubble size estimation is of great importance when considering the membrane-cleaning efficiency, the applicability of an available correlation for bubble diameter is examined. Davidson and Schuler ${ }^{(12)}$ proposed the following correlation:

$$
d=1.38\left(\frac{Q^{2}}{g}\right)^{0.2}
$$

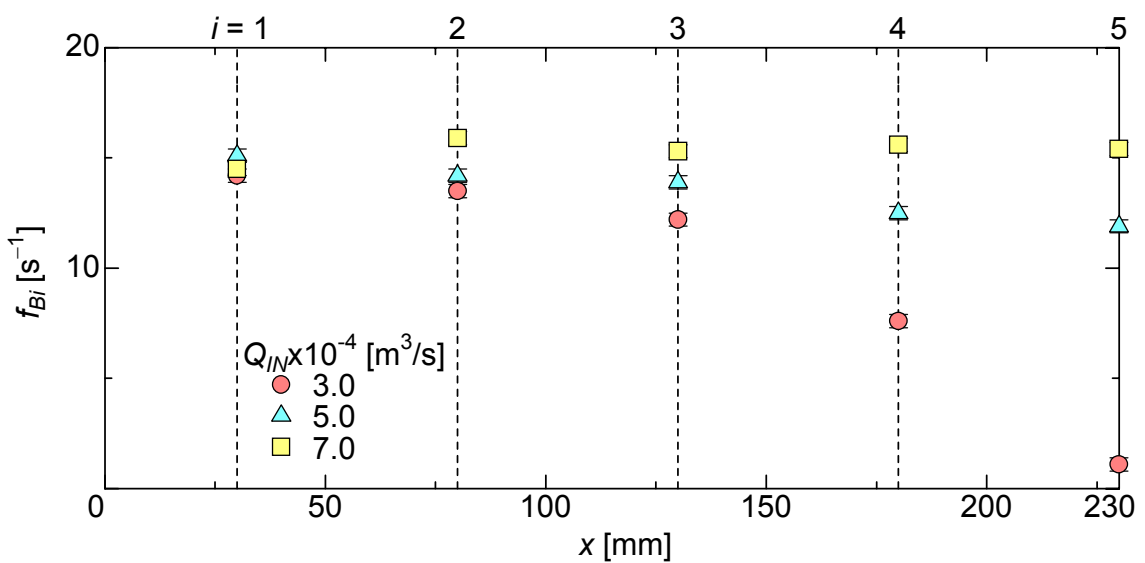

(a)

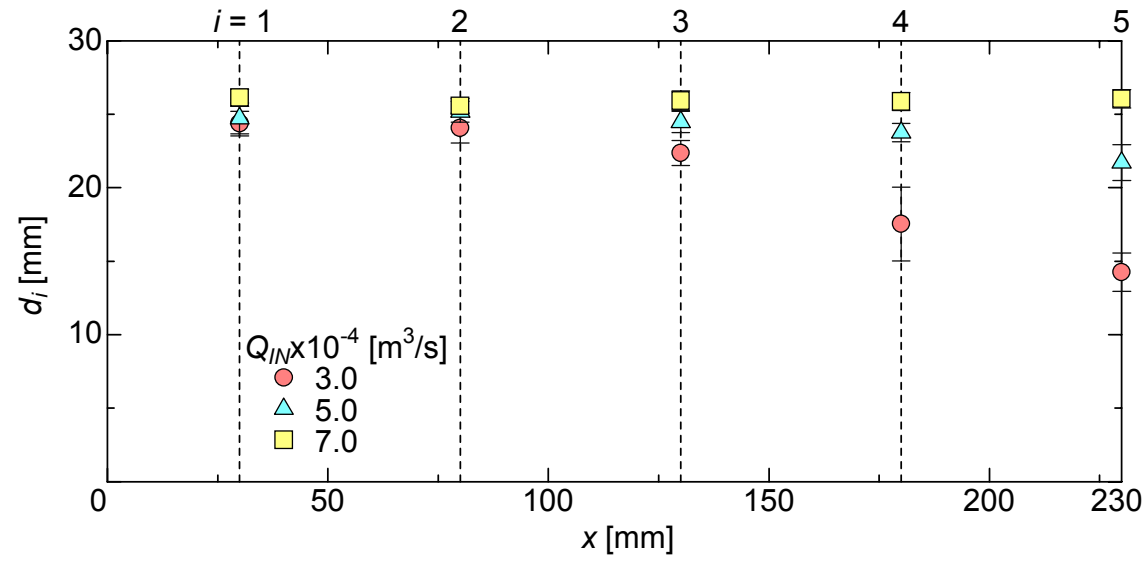

(b)

Fig. 10 Bubble generation frequency, $f_{B i}$, (a) and bubble diameter, $d_{i}$ (b) 


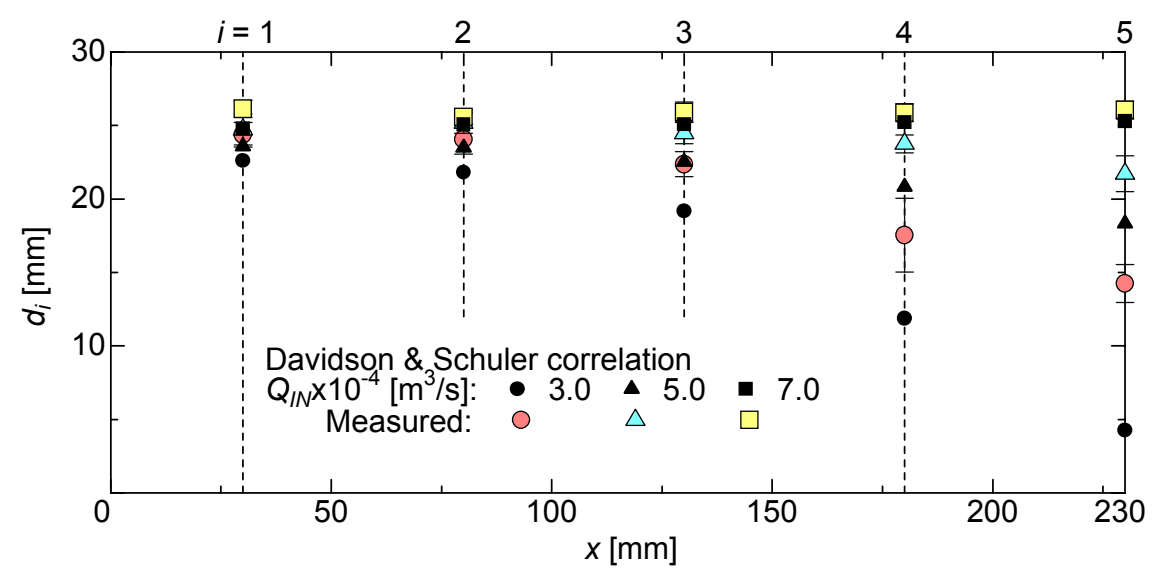

Fig. 11 Comparison of $d_{i}$ between measured data and the Davidson-Schuler correlation $\left(D_{H}=5 \mathrm{~mm}\right)$

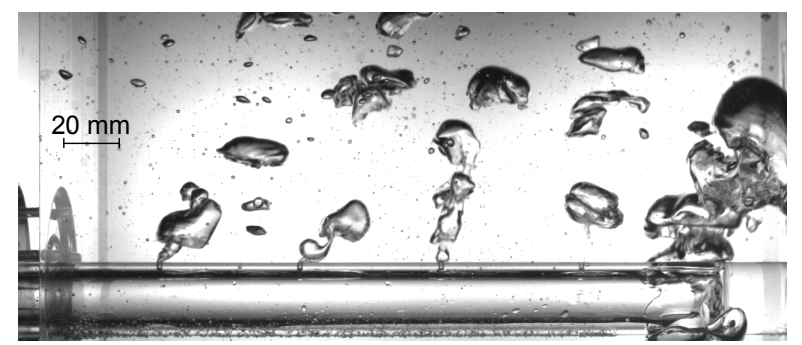

(a) $D_{H}=2 \mathrm{~mm}$

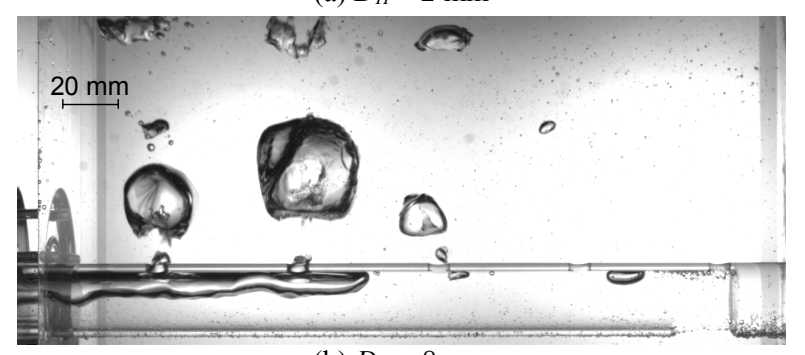

(b) $D_{H}=8 \mathrm{~mm}$

Fig. 12 Flows at $Q_{I N}=5.0 \times 10^{-4} \mathrm{~m}^{3} / \mathrm{s}\left(D_{H}=2\right.$ and $\left.8 \mathrm{~mm}\right)$

where $d$ is the diameter of a bubble generated from a single orifice at a constant flow rate, $Q$. Although this correlation was proposed for bubbles generated from a single orifice, Maekawa et al. ${ }^{(13)}$ confirmed that this correlation is also applicable to bubbles generated from a diffuser plate having several tens of aeration holes at constant flow rates. Substituting measured $Q_{i}$ into $Q$ in Eq. (3) gives $d_{i}$ shown in Fig. 11. The correlation gives reasonable estimations. The agreement becomes better as $Q_{I N}$ increases. The difference between the measured and calculated $d_{i}$ is, however, large for $i=4$ and 5 at $Q_{I N}=3.0 \times 10^{-4}$ and $5.0 \times 10^{-4} \mathrm{~m}^{3} / \mathrm{s}$ since the flow rates at these holes were not constant due to slugging. The correlation is therefore applicable to evaluation of $d_{i}$, provided that the constant flow rate condition is satisfied.

\subsection{Effects of $D_{H}$ on $Q_{i}$}

Effects of the hole diameter on the gas flow rate were investigated. Figure 12 (a) shows a flow in the pipe of $D_{H}=2 \mathrm{~mm}$ at $Q_{I N}=5.0 \times 10^{-3} \mathrm{~m}^{3} / \mathrm{s}$. The small $D_{H}$ decreases the flow rate from each aeration hole and the residual air flows out from the bottom hole. The small $D_{H}$ also makes the water level very low. To the contrary, in the flow with the larger $D_{H}\left(D_{H}\right.$ $=8 \mathrm{~mm}$ ), most of air flows out from the holes $i=1$ and 2 and the holes $i=3,4$ and 5 are filled with water as shown in Fig. 12 (b). Figure 13 shows a comparison of $Q_{i}$ between $D_{H}=$ 2,5 and $8 \mathrm{~mm}$. The flow rate from $i=5$ for $D_{H}=2 \mathrm{~mm}$ was not measurable due to the bubble generation from the bottom hole. This comparison clearly shows that $D_{H}$ strongly 
affects $Q_{i}$. According to the orifice equation and the Terasaka-Tsuge correlation for the orifice coefficient, $k=0.813 D_{H}^{2}$, the gas flow rate from the single orifice is proportional to $D_{H}{ }^{2}$ for a given pressure difference, $\left|P_{G i}-P_{B i}\right|$. This qualitatively explains the reason why $Q_{i}$ increases with $D_{H}$ at small $i$ in the present experiment.

The Davidson-Schuler correlation also gives good predictions of $d_{i}$ at the aeration holes which are almost free from the gas flow disturbance due to the slugging, i.e., the holes $i=$ 1-4 for $D_{H}=2 \mathrm{~mm}$ and $i=1$ and 2 for $D_{H}=8 \mathrm{~mm}$. The bubble diameter, therefore, can be correlated in terms of $Q_{i}$ only.

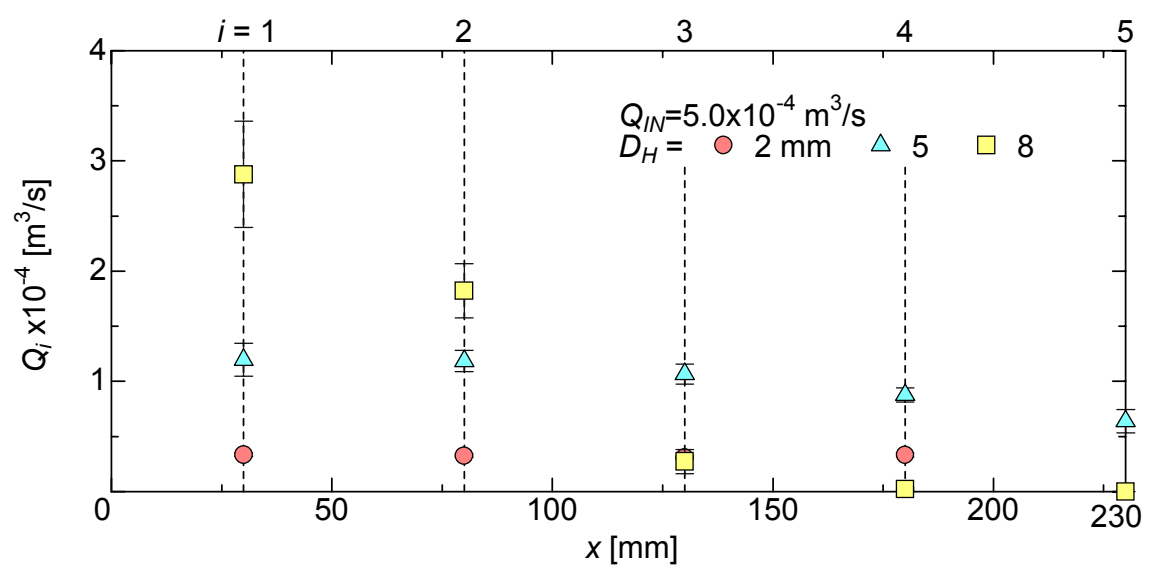

Fig. 13 Flow rates for $D_{H}=2$ and $8 \mathrm{~mm}$

\section{Conclusion}

Effects of the total gas flow rate on the flow rates from aeration holes and water level in a diffuser pipe for a membrane bioreactor were experimentally investigated. The applicability of a correlation for bubble diameter proposed by Davidson and Schuler was also examined. The conclusions obtained are as follows:

(1) The gas flow rate from the aeration hole depends on the water level inside the diffuser and becomes constant for all the holes as the total gas flow rate increases since the high total gas flow rate make the water level uniform, which results in a constant gas pressure in the diffuser.

(2) The onset of slugging in the diffuser pipe is well correlated in terms of the local gas velocity and the Mishima-Ishii's slugging model.

(3) The increase in the total gas flow rate decreases the water level, causing suppression of the onset of slugging.

(4) The diameter of aeration hole strongly affects the gas flow rate from each aeration hole and water level.

(5) The Davidson-Schuler correlation gives reasonable estimations of the bubble diameter, provided that the influence of slugging is not significant.

\section{Acknowledgement}

The authors would like to express their thank to Mr. Yusuke Fujimoto for his assistance in the experiments. 


\section{References}

(1) Smith, C. W., Di Gregorio, D., Talcott, R. M., The Use of Ultrafiltration Membrane for Activated Sludge Separation, In Proceedings of the 24th Annual Purdue Industrial Waste Conference, pp. 1300-1310 (1969)

(2) Brindle, K., Stephenson, T., The Application of Membrane Biological Reactors for the Treatment of Wastewaters, Biotechnology and Bioengineering, 49, pp. 601-610 (1996)

(3) Pabby, A., Rizvi, S. H., Sastre, A. (eds.), Handbook of Membrane Separations, CRC Press (2009)

(4) Drews, A., Membrane Fouling in Membrane Bioreactors - Characterization, Contradictions, Cause and Cures, Journal of Membrane Science, 363, pp. 1-28 (2010)

(5) Fenu, A., Roels, J., Wambecq, T., De Gussem, K., Thoeye, C., De Gueldre, G., Van De Steene, B., Energy Audit of a Full Scale MBR System, Desalination, 262, pp. 121-128 (2010)

(6) Ozdemir, B., Saatci, A., Yenigun, Evaluation of Cake Filtration Biological Reactors (CFBR) vs. Membrane Biological Reactors (MBR) in a Pilot Scale Plant, Desalination, 288, pp. 135-144 (2012)

(7) Takashima, T., Itakura, M., Kinoshita, I., Activated Sludge Processing Unit, Japanese Patent Disclosure P4431682 (2010)

(8) Kawagishi, T., Yanone, K., Nakahara, Y., Operating Method of Aeration Equipment, Japanese Patent Disclosure 2011-92835 (2011)

(9) Russ, J. C., The Image Processing Handbook, 6th ed., CRC Press, (2011)

(10) Mishima, K., Ishii, M., Theoretical Prediction of Onset of Horizontal Slug Flow, Trans. ASME, J. Fluid Eng., 102, pp. 441 (1980)

(11) Terasaka, K., Tsuge, H., Bubble Formation at a Single Orifice in Highly Viscous Liquids, Journal of Chemical Engineering of Japan, 23(2), pp. 160-165 (1990)

(12) Davidson, J. F., Schuler, B. O. G., Bubble Formation at an Orifice in an Inviscid Liquid, Transaction of the Institution of Chemical Engineers, 38, pp. 335-342 (1960)

(13) Maekawa, M., Sou, A., Hosokawa, S., Tomiyama, A., Effects of Inlet Bubble Diameter on Bubbly Flow in a Bubble Column under High Gas Volume Flux Condition, Trans. JSME, Ser. B., 74(742), pp. 1368-1375 (2008) 